\title{
RETINOPATÍA DE LA PREMATURIDAD: PRIMERA CAUSA DE CEGUERA INFANTIL. PROBLEMÁTICA Y TRATAMIENTO
}

\author{
Augusto Chafloque Cervantes ${ }^{1}$, Fabiola Quezada Baltodano ${ }^{2}$, Giovanni F. Rivera Arce ${ }^{3}$, \\ Evelyn Aspajo Tejada ${ }^{4}$
}

\begin{abstract}
RESUMEN
La retinopatía de la prematuridad constituye la primera causa de ceguera infantil en el Perú y en muchos países cuya tasa de sobrevida en el recién nacido de muy bajo peso al nacer se ha incrementado, esto debido, al mayor apoyo biotecnológico en el manejo de cuidados intensivos neonatales. Se considera pertinente revisar los aspectos referentes a la fisiopatología, etiopatogenia multifactorial y epidemiologia de esta enfermedad. Asimismo se enfatizan los métodos de tamizaje utilizados con los factores de riesgo asociados, la clasificación internacional en el diagnóstico y los tratamientos actuales y experimentales basados en trabajos multicentricos con las recomendaciones respectivas. Finalmente se exponen las patológicas oculares con riesgo incrementado que pueden padecer aquellos neonatos con antecedente de retinopatía de la prematuridad.
\end{abstract}

Palabras clave: Retinopatía de la prematuridad; Factores de riesgo; Tamizaje; Tratamientos (Fuente: DeCS BIREME).

\section{RETINOPATHY OF PREMATURITY: LEADING CAUSE OF CHILDHOOD BLINDNESS. PROBLEMS AND TREATMENT}

\begin{abstract}
Retinopathy of prematurity is the leading cause of childhood blindness in Peru and in many countries whose survival rate in newborns of very low birth weight has increased, this due to the largest biotech support in the management of intensive care neonatal. It is considered appropriate to review the aspects related to the pathophysiology, multifactorial etiology and epidemiology of this disease. Screening methods used with associated risk factors, the international classification in the diagnosis and current and experimental treatments based on multicenter studies with the respective recommendations are also emphasized. Finally pathological eye is exposed to increased risk of suffering those infants with a history of retinopathy of prematurity.
\end{abstract}

Key words: Retinopathy of prematurity; risk factors; Screening; Treatment (Source: MeSH NLM).

\section{INTRODUCCIÓN}

La retinopatía del prematuro es una patología de la modernidad. Recién a mediados del siglo pasado, cuando comenzaron a sobrevivir prematuros en grandes números, Terry describió la "fibroplasia Retrolental", ceguera incurable generalmente bilateral que afectaba a niños prematuros, la cual se asoció inicialmente a la administración de oxígeno. El nombre describe los hallazgos en los ojos de estos niños ciegos en que se observaba una membrana blanquecina fibrosa por detrás del cristalino, correspondiente a la retina totalmente desprendida. Esto impulsó el desarrollo de tecnologías para monitorizar las necesidades de $\mathrm{O} 2$ y su administración a los prematuros tratando de encontrar niveles de oxigenación adecuados, pero seguros. En los años 60 , la invención del oftalmoscopio binocular indirecto permitió observar por primera vez las fases iniciales de la enfermedad que ocurrían en la periferia retinal y entonces se cambió su nombre a retinopatía del prematuro. La segunda epidemia sobrevino en los años 70 , cuando los métodos de monitorización neonatal permitieron la sobrevida de prematuros cada vez más pequeños y entonces volvió a aparecer esta ceguera, pero ahora sin una asociación causal directa con la administración de O2, sino con la prematuridad en sí. En los años 80 , aun no existía un tratamiento para esta enfermedad que dejaba cada vez más niños ciegos en los países desarrollados. Con gran esfuerzo, se desarrolló una clasificación internacional (ICROP) basada en la morfología observable en el fondo de ojo con oftalmoscopia binocular indirecta (la cual permite visualizar la retina hasta sus áreas más periféricas) y así ver las etapas precoces de la enfermedad ${ }^{1}$. Esta clasificación permitió realizar un ensayo clínico multicéntrico controlado, pionero en oftalmología, el CryoROP. En él se randomizó los ojos que tuviesen alrededor de un $50 \%$ de riesgo de quedar ciegos basados en lo que se conocía hasta entonces sobre la historia natural de la enfermedad. Como la enfermedad es generalmente bilateral y simétrica el otro ojo servía de control. El tratamiento, en el cual se aplicaba crioterapia transescleral a toda la retina avascular del ojo (la más periférica), disminuyó a la mitad los casos de ceguera de un $50 \%$ a un $25 \%{ }^{2}$.

\footnotetext{
Jefe del servicio de Oftalmología Pediatría del Instituto Nacional Materno Perinatal. Lima - Perú

Oftalmólogo Asistente del Instituto de Ojos Oftalmosalud. Lima - Perú

Oftalmólogo Asistente del Hospital Regional Honorio Delgado. Arequipa -Perú

Optómetra del Instituto Nacional Materno Perinatal. Lima - Perú
} 


\section{DIAGNÓSTICO}

La clave en la prevención de la ceguera por ROP es el examen seriado de la retina bajo dilatación pupilar con oftalmoscopia binocular indirecta a todos los prematuros en riesgo, a partir de las 4 a 6 semanas de vida ${ }^{3}$. Inicialmente se observa la inmadurez de los vasos de la retina que no llegan hasta el final de ésta, dejándola avascular en la periferia. Cuando los vasos retinales se detienen en una línea visible abrupta que luego se engruesa (bridge), estamos frente a las etapas leves de la retinopatía que tienen un $90 \%$ y $75 \%$ de posibilidad de mejorarse espontáneamente. Recién cuando comienza a observarse aparición de neovasos anómalos en este bridge y se dilatan y ensortijan los vasos del polo posterior del ojo es necesario intervenir con un tratamiento. Los pesos de nacimiento y edades gestacionales bajo los cuales es necesario examinar a los prematuros dependen de la calidad del cuidado neonatal, a mejor cuidado neonatal posible ir bajando los criterios de selección ${ }^{4}$. En el Perú actualmente se recomienda examinar a todos aquellos niños que pesen menos de 1500 gramos al nacer y/o menores de 32 semanas de edad gestacional. Los exámenes oftalmoscópicos seriados deben continuarse hasta verificar que se ha completado la maduración retinal, sólo ahí el riesgo de retinopatía desaparece ${ }^{5}$. El Cryo-ROP demostró la utilidad del tratamiento, pero aun así el porcentaje de ojos que quedaban ciegos era alto (25\% inicialmente y mayor en seguimientos a más largo plazo) ${ }^{6}$. Por esto, se diseñó un nuevo estudio multicéntrico, ahora con láser (previamente no disponible), para evaluar la conveniencia de intervenir más precozmente en el desarrollo de la enfermedad y así mejorar los resultados. Este trabajo multicéntrico controlado y randomizado para tratamiento precoz (EarlyTreatment ET-ROP) eligió para la intervención terapéutica el momento en que el riesgo de ceguera para el ojo fuese de $15 \%{ }^{7}$. Este riesgo era posible calcularlo para cada ojo con un programa computacional diseñado a partir de los datos y resultados de historia natural (ojos control) del Cryo-ROP. El estudio demostró la utilidad de tratar precozmente a un subgrupo de casos del total, caracterizados por sus hallazgos morfológicos específicos al fondo de ojo lo que se denominó Retinopatía Tipo1. Esta se caracteriza por ubicarse más posteriormente en el fondo de ojo, es decir más cerca de la mácula y con mayor área total de retina avascular, tener mayor compromiso vascular con dilatación y tortuosidad de los vasos del polo posterior (enfermedad plus) y progresar en forma más rápida. La revisión de la clasificación Internacional (IC-ROP revisited) describió también una forma especialmente agresiva de la enfermedad denominándola AP-ROP (Aggressive Posterior ROP) la cual constituye un subgrupo de la Retinopatía tipo 1, de peor pronóstico,que ha aumentado en incidencia en los últimos años ${ }^{8}$. EI ET-ROP mostró la utilidad de intervenir más precozmente en la evolución de la enfermedad en un subgrupo de pacientes de alto riesgo (Retinopatía tipo 1). Aun así, a pesar de la efectividad aumentada del ET-ROP con respecto al Cryo-ROP y siguiendo todos los protocolos recomendados hay aproximadamente un $10 \%$ de ojos en los cuales la enfermedad lleva a mala visión, incluso a pesar del tratamiento adecuado y a tiempo, y evolucionan al desprendimiento retinal y la ceguera de sus ojos.

\section{EPIDEMIOLOGÍA}

Hoy en día la mayor incidencia de ceguera por retinopatía del prematuro se encuentra en los países en vías de desarrollo como Perú ${ }^{4}$. En los países desarrollados los niños prematuros sobreviven exitosamente debido al excelente cuidado neonatal; además existen los controles oftalmológicos oportunos y adecuados para prevenir la ceguera, con el tratamiento a tiempo en la gran mayoría de los casos (aunque no en todos como vimos). De hecho, en los países desarrollados la primera causa de déficit visual en niños prematuros no es la ROP sino el déficit visual cerebral, es decir, daño neurológico. Los países pobres, en el otro extremo, no tienen la calidad de cuidado neonatal como para que los prematuros sobrevivan y por lo tanto no se enfrentan a este problema.Es en los países latinoamericanos donde la ROP es la primera entre las causas de ceguera infantil, ya que el cuidado neonatal existe y permite que los prematuros sobrevivan, pero no siempre hay una red oftalmológica preparada para examinar y tratar a tiempo a estos niños. Una situación similar se avizora pronto para India y China al mejorar su cuidado neonatal. En el manejo de la ROP es clave la existencia de una ventana de oportunidad terapéutica que generalmente se extiende entre las $36 \mathrm{y}$ las 44 semanas de edad gestacional corregida. Esta es una actitud que en nuestro país se aplicó, con la elaboración de una Guía Clínica de ROP por expertos en el tema y aprobada por el MINSA $^{5}$ en el 2006 y la Norma técnica de atención del recién nacido pre termino con riesgo de Retinopatía del Prematuro publicada en el 2010.

\section{FISIOPATOLOGÍA}

La enfermedad se produce porque al nacimiento los vasos retinales no están plenamente desarrollados, han avanzado solo parcialmente desde el nervio óptico hacia la periferia retinal. La retina periférica, por lo tanto, no tiene vasos propios, pero al ser delgada e inmadura se oxigena adecuadamente desde la coroides subyacente. El alza relativa en las presiones parciales de $\mathrm{O} 2$ que se produce en el ambiente extrauterino detiene el avance de la vascularización propiamente retinal ${ }^{9}$. Al comenzar la retinopatía se observa una clara línea que delimita la retina vascular (más posterior) de la avascular (más periférica). Una vez que la retina periférica se diferencia y madura aumenta en grosor, aumentan entonces sus requerimientos y no le basta el $\mathrm{O} 2$ que difunde desde los vasos coroideos por lo que se genera una hipoxia relativa en estas células, que comienzan a fabricar factor de crecimiento vascular endotelial(VEGF), el cual estimula el re-crecimiento de los vasos retinales. Estos pueden 
retomar su camino normal avanzando por la retina hacia la periferia, en cuyo caso observamos una mejoría espontánea de la enfermedad. Pero una minoría, un porcentaje pequeño de niños, aquellos más prematuros, no logran reiniciar este proceso fisiológicamente y los vasos retinales estimulados a crecer por el VEGF no lo hacen avanzando hacia la retina avascular sino que entran haciael vítreo perpendicularmente al plano de laretina. Este es el momento clave, el de la neovascularización patológica, donde los vasos anormales emergen desde un "bridge" (levantamiento retinal) ubicado en el límite entre retina vascular y avascular y se introducen en el vítreo ${ }^{1}$.

\section{TRATAMIENTO ACTUAL}

Consiste en quemar con múltiples disparos de láser indirecto a través de la pupila dilatada, la retina avascular periférica. Se indica cuando el examen morfológico del fondo de ojo muestra signos evidentes de neovascularización anormal, según los criterios del ET-ROP: una Retinopatía Tipo 1, pero sin que se haya producido aún desprendimiento retinal ${ }^{7}$. Con este tratamiento se destruyen la gran mayoría de las células hipóxicas de la retina avascular, lo que disminuye su producción del factor de crecimiento vascular endotelial (VEGF), factor principal entre los que promueven la neovascularización. La meta de este tratamiento es preservar la mácula, ubicada en el centro de la retina, donde reside la habilidad para discriminar detalles finos (la agudeza visual propiamente tal) por su alta concentración de fotorreceptores, lo que nos permite leer, por ejemplo. Sin el tratamiento, la retina periférica también se perdería funcionalmente debido al desprendimiento retinal total. La pérdida de campo visual periférico que se observa asociada al tratamiento con láseres es relativa, ya que se ha demostrado que incluso niños que han tenido retinopatía severa y que no fueron tratados, se recuperaron espontáneamente, y sin embargo, perdieron también en algún grado sus campos visuales periféricos ${ }^{6}$. Por otro lado, normalmente, la retina más periférica sólo tiene un rol marginal en el campo visual y en la función visual integral. A pesar del tratamiento oportuno con láser, un 9 a $14 \%$ de los pacientes tratados evolucionan al desprendimiento de retina con ceguera. A más largo plazo los resultados son aún más desalentadores, a los 6 años de edad, un $65 \%$ de los ojos tratados con Retinopatía tipo1 en el ET-ROP tienen una visión peor que 20/40. No son necesariamente ciegos, pero sólo un $35 \%$ tiene visión normal ${ }^{10}$.

\section{TRATAMIENTOS EN EXPERIMENTACIÓN}

En los casos más graves, que no muestran disminución de la retinopatía en los días subsecuentes al tratamiento con láser, se puede intentarla inyección de anticuerpos anti-VEGF directamente en el vítreo. Este tratamiento experimental aparece como muy prometedor, con reportes de casos con buenos resultados. El uso de anticuerpos anti-VEFG que es de rutina en otras patologías oculares como la maculopatía relacionada con la edad, es aún controversial en la ROP porque el VEGF cumple multiplicidad de funciones en los tejidos en desarrollo que podrían ser interferidas con potencial iatrogenia ${ }^{11}$. De gran relevancia es el efecto neuroprotector del VEGF sobre las células de la retina que las protege contra el daño isquémico. EI VEFG tiene además un rol clave en el desarrollo de los vasos más allá de la retina, regula la angiogénesis en otros órganos incluido el SNC y los pulmones. Críticamente está activo en otros tejidos justo en el período en que se administra su anticuerpo en forma intravítrea. La "enfermedad plus" existente dentro del ojo en estos casos evidencia una rotura de la barrera hematoretinal que permitiría su mayor difusión sistémica desde el sitio de inyección intraocular. Por todo esto es necesario ser muy cautos en su utilización. Lo similar en ambos casos fue la introducción de tratamientos para lograr beneficios a corto plazo sin considerar sus posibles efectos deletéreos en el largo plazo. A pesar del éxito que están teniendo los agentes anti VEGF en ROP debemos ser extremadamente cautos antes de introducir una terapia potencialmente dañina, cuando existe una terapia con efectividad comprobada, aunque limitada, como el láser. Por último, en aquellos casos en que sobreviene el desprendimiento retinal, el único tratamiento disponible es la vitrectomía para intentar reaplicar la retina. Este tratamiento a veces logra resultados anatómicos favorables, pero muy pocas veces permite recuperar función visual significativa.

Los resultados del trabajo publicado en el 2011 del Bevacizumab Eliminates the Angiogenic Threat of Retinopathy of Prematurity (BEAT-ROP) concluyo lo siguiente: 1.- Bevacizumab es superior que el láser para el tratamiento de ROP 3 en zona I con enfermedad plus, 2.- La vascularización periférica retiniana continua normalmente en el grupo de tratamiento con bevacizumab a diferencia del grupo de tratamiento con láser, y 3.Bevacizumab es un medicamento poco costoso que puede ser administrado rápidamente en una cuna por cualquier oftalmólogo entrenado ${ }^{12}$.

Recientemente, la Asociación Americana de Pediatría (AAP), la Asociación Americana de Oftalmología (AAO), la Asociación Americana de Oftalmología Pediátrica y Estrabismo (AAPOS) y la Asociación Americana de Ortoptistas Certificados (AACO) revisaron la guía de tamizaje y tratamiento de neonatos prematuros para retinopatía de la prematuridad publicada en 2006 y han recomendado en la guía del 2013 considerar el uso de bevacizumab para el tratamiento de ROP 3 en zona I con enfermedad plus incluso a pesar que bevacizumab no se encuentra aprobado actualmente por la FDA para el tratamiento del ROP ${ }^{13}$.

\section{MÉTODOS DE TAMIZAJE}

Diagnosticar la ROP a tiempo es un gran problema, incluso para los países desarrollados: hay que contar con suficientes oftalmólogos calificados y motivados para 
realizar el examen seriado de los niños prematuros en riesgo en las unidades de neonatología que muchas veces no están cerca de los centros oftalmológicos. El examen de oftalmoscopia binocular indirecta en prematuros (el "Gold Standard" actual) requiere de gran experiencia aún entre oftalmólogos ${ }^{14}$. La Retcam (cámara fotográfica de contacto corneal especialmente diseñada para prematuros) es el principal entre estos métodos con resultados promisorios, pero su costo desafortunadamente es aún prohibitivo para países en vías de desarrollo donde es especialmente álgido el problema. Otros métodos fotográficos con equipos más baratos (cámara de fondo no de contacto) están en evaluación, pero aún no han sido avalados con sensibilidad y especificidad suficientes ${ }^{15}$. Uno de los mayores problemas es la gran cantidad de niños que deben ser examinados para detectar sólo unos pocos casos en verdadero riesgo de ceguera y que necesitan tratamiento. Es muy alentador la aparición reciente de programas computacionales (WINROP) que a través de la ganancia de peso del niño en las primeras semanas de vida son capaces de predecir con mayor exactitud quienes están en riesgo, de ROP permitiendo disminuir el universo de niños a examinar a un cuarto del total actual ${ }^{16}$.Esto permitiría aliviar la carga de exámenes diagnósticos en forma significativa, sin perder los casos en verdadero riesgo. Esta metodología aún se encuentran en etapa de evaluación a nivel internacional ${ }^{17}$

\section{OTROS EFECTOS DE LA PREMATURIDAD SOBRE LA VISIÓN}

Si bien la ROP es el más grave de los riesgos que corren los ojos de los prematuros, no es desafortunadamente el único. Aquellos prematuros que logran mejorarse ya sea espontáneamente o con tratamiento de su ROP, e incluso algunos que no han desarrollado ROP presentan mayor riesgo visual por muchos años, por su mayor incidencia de miopía, estrabismo y déficit visual cerebral.

\section{MIOPÍA}

La miopía de los prematuros suele aparecer precozmente y puede ser severa. Se diferencia característicamente de la miopía habitual de los niños por su inicio muy precoz. Se detecta en algunos casos desde los primeros meses de vida y los casos severos con más de 6 dioptrías de miopía van en aumento progresivo hasta los 3 años de edad por lo menos ${ }^{18}$. La incidencia global de miopía aumenta al bajar el peso de nacimiento y también progresivamente con la mayor gravedad de ROP alcanzada durante la evolución aguda de la enfermedad. Va disminuyendo a mayor Peso de Nacimiento (PN) y Edad Gestacional (EG) al nacer. La incidencia general en todos los prematuros extremos es de alrededor de 30\%. La miopía de los prematuros debe corregirse con anteojos cuando su magnitud interfiere con un adecuado desarrollo sicomotor para la edad. La causa de la miopía en los prematuros es principalmente un cristalino demasiado grueso (con mucho poder dióptrico), lo cual es una característica del ojo prematuro. Es como si la maduración global del ojo con adelgazamiento del cristalino postnatal no se produjese adecuadamente en estos casos. Puede haber también un aumento del largo axial del ojo que contribuye a la miopía en algunos casos, pero es lo menos frecuente. Este aumento de la potencia refractiva del ojo, más que el largo excesivo, se ha verificado también en adultos miopes que fueron prematuros menores de 32 semanas al nacer, y presentan un aumento de la curvatura corneal. Con esto vemos que la miopía asociada a la prematuridad permanece incluso hasta la vida adulta, presentando características refractivas propias ${ }^{19}$. Fielder y Quinn han propuesto la teoría de que las lesiones de la retinopatía causan una restricción mecánica al crecimiento ocular en la parte posterior del globo que es donde hay mayor desarrollo en la etapa tardía fetal y precoz postnatal ${ }^{20}$. Esta inhibición relativa del crecimiento dejaría un largo axial más corto pero también causaría una restricción en el desarrollo del polo anterior determinando inicialmente grosores cristalinéanos excesivos y curvaturas cornéales aumentadas con la subsecuente miopía.

\section{ANISOMETROPÍA}

Además de tener miopía, un $30 \%$ de los prematuros tiene anisometropía. La anisometropía consiste en tener un poder refractivo diferente entre ambos ojos. Esto es especialmente peligroso ya que el ojo más miope tiende a ambliopizarse lo que significa perder la AV potencial máxima del ojo, durante la infancia porque el cerebro escoge utilizar el ojo menos miope con el que ve mejor y suprime la imagen desenfocada del ojo más miope, generándose así una pérdida de agudeza visual por no uso del ojo afectado. Esta pérdida de agudeza visual se convierte en irreversible pasados los 9 años de edad. Es necesario tomar la agudeza visual a los niños prematuros a lo largo de la infancia para poder detectar peor visión en un ojo que el otro. Si la miopía en el ojo mejor es pequeña, el defecto del ojo peor puede pasar totalmente desapercibido hasta que es muy tarde para corregirlo. La anisometropía obliga a indicar lentes ópticos a los niños afectados para prevenir la ambliopía, y si esta ya se ha producido en algún grado, está indicado además parchar el mejor ojo diariamente por algunas horas para recuperarla.

\section{ASTIGMATISMO}

El astigmatismo significativo es también más frecuente en los prematuros que en los niños de término, presentándose hasta un $40 \%$ de los casos, más si tuvieron ROP y más mientras más grave fue ésta ${ }^{21}$. El astigmatismo significativo produce mucho desenfoque de la imagen retinal por lo que también requiere la corrección con lentes ópticos en la infancia para prevenir la ambliopía en la cual el ojo nunca aprende a ver realmente nítido porque 
nunca se enfrenta a imágenes lo suficientemente claras como para entrenarse en esa tarea de discriminación fina. ESTRABISMO El estrabismo también es más frecuente en prematuros que en niños de término. El seguimiento de la cohorte del ET - ROP encontró un $20 \%$ a los 6 meses y $30 \%$ a los 9 meses entre los con retinopatía de alto riesgo. En los con ROP de bajo riesgo bajaba a $10 \%{ }^{22}$.Generalmente se trata de una endodesviación o estrabismo convergente de magnitud variable que tarda más en estabilizarse en el tiempo, pero también pueden observarse otras desviaciones. Peor agudeza visual en un ojo que en el otro es un factor de riesgo, ya sea causada por asimetría de la visión por secuelas de la retinopatía o por anisometropía. Con los años la desviación tiende a ir disminuyendo espontáneamente, y puede incluso convertirse en una exodesviación o estrabismo divergente. En general no tiene buen resultado quirúrgico por su misma inestabilidad y por la gran facilidad de sobre corregirse eventualmente a largo plazo. La principal alteración visual que produce este estrabismo no es visión doble (diplopía) como en el adulto, sino que ausencia de desarrollo de la visión binocular al no utilizar el cerebro ambos ojos simultáneamente. Además de esto, existe el riesgo de ambliopía del ojo desviado con mayor frecuencia ya que el cerebro tiende a suprimir esta imagen. Para enfrentar el riesgo de ambliopía en estos niños es imprescindible mantenerlos bajo un control oftalmológico seriado durante la infancia en el cual se corrijan con lentes los vicios de refracción (miopía, astigmatismo y anisometropía) y se combata la ambliopía con parche diario por algunas horas al ojo de mejor agudeza visual a fin de estimular la visión de ojo más débil. En los niños con parálisis cerebral o con leucoma lacia periventricular la incidencia de estrabismo llega al $50 \%$. Su incidencia va aumentando además al bajar el peso de nacimiento. La parálisis cerebral en prematuros tiene una fisiopatología diferente a la de los niños de término. En prematuros, la región periventricular, incluyendo las radiaciones ópticas,es una divisoria de aguas de la irrigación cerebral por lo que es especialmente sensible al daño hipóxico, generándose como consecuencia de éste una leucomalacia periventricular que afecta principalmente a la substancia blanca. Esto aumenta el riesgo de que tengan déficit visual cerebral (Cerebral Visual Impairment CVI) radicado en esta zona, más que en la corteza visual. En países desarrollados, en los cuales se ha logrado una adecuada prevención de la ceguera por ROP, es este CVI la principal causa de déficit visual en prematuros, sobrepasando incluso a los casos de ROP con tratamiento fallido.

\section{OTRAS ALTERACIONES}

La atrofia óptica por hipoxia también puede verse en prematurospero no se da aisladamente sino que generalmente con PC, epilepsia y retardo del desarrollo sicomotor asociado. Las hemorragias intraventriculares que frecuentemente se observan en prematuros provienen de los frágiles capilares de la matriz germinal subependimaria, muy susceptibles a cambios de la presión arterial ${ }^{23}$. Estos eventos pueden determinar un hidrocéfalo post-hemorrágico cuyos signos oftalmológicos son la parálisis de la mirada superior y la retracción del párpado superior en el signo del "sol poniente". Daños más sutiles de la función visual asociados a la prematuridad pueden ser especialmente difíciles de detectar clínicamente. Estos incluyen: disminución de la sensibilidad al contraste, pérdidas de campo visual y alteración en la visión de colores ${ }^{24}$.

\section{CONCLUSIÓN}

La gran repercusión que puede tener la prematuridad sobre la función visual obliga a prestar cuidadosa atención al desarrollo visual de estos niños. El diagnóstico y tratamiento precoz de la retinopatía del prematuro es un imperativo en el período neonatal ya que no existe intervención más costo efectiva que ésta en toda la oftalmología para disminuir años de ceguera. Una vez sorteado este escollo, los prematuros menores de 1500 gramos al nacer deben continuar bajo control oftalmológico seriado para detectar a tiempo la miopía, anisometropía, astigmatismo y estrabismo que les son característicos y realizar el tratamiento de la ambliopía asociada a ellos. Se recomienda al menos un control anual hasta los cinco años

\section{REFERENCIAS BIBLIOGRÁFICAS}

1. Committee for the Classification of Retinopathy of Prematurity. international classification of retinopathy of prematurity. Arch Ophthalmol 1984;102(8):1130-1134.

2. Cryoterapy for Retinopathy of Prematurity Cooperative group. Multicenter trial of Retinopathy of Prematurity: Preliminary results. Arch Ophthalmol 1988;106:471-479.

3. Committee for the Section on Ophthalmology, American Academy of Pediatrics; American Academy of Ophthalmology; American Association for Pediatric Ophthalmology and Strabismus. Screening examination of premature infants for retinopathy of prematurity Pediatrics 2006;118(3):1324.

4. Reynolds JD, Dobson V, Quinn GE, Fielder AR, Palmer EA. Evidence-based screening criteria for retinopathy of prematurity: natural history data from the CRYO-ROP and LIGHT-ROP studies. ArchOphthalmology. 2004; 120.

5. Guía clínica Retinopatía del prematuro. Ministerio de Salud de Perú. 2006.

6. Cryoterapy for Retinopathy of Prematurity Cooperative group. Multicenter trial of Retinopathy of Prematurity: Ophthalmological outcomes at 10 years. Arch Ophthalmol 2001;119:1110-1118.

7. Early Treatment for Retinopathy of Prematurity Cooperative Group. Revised indications for the treatment of retinopathy of prematurity: results of the Early Treatment for Retinopathy of Prematurity randomized trial. Arch Ophthalmol. 2003;121(12):1684- 1694.

8. An International Committee for the Classification of Retinopathy of Prematurity. The International Classificatioj of Retinopathy of Prematurity.Revisited. Arch Ophthalmol 2005; 123:991-999.

9. Smith LEH. Through The Eyes of a Child: Understanding Retinopathy through ROP: The Friedenwald Lecture. IOVS 200; 49(12): 5177-5182. 
10. The Early treatment of Retinopathy Cooperative Group. Final visual acuity results in the Early Treatment for Retinopathy of Prematurity Study. Archives Ophthamol 2010;128(6):663-671.

11. Darlow BA, Gilbert C. Quinn GE, Azad R, Ells A, Fielder A, Zin A Promise and potencial pitfalls of anti VEGF drugs in Retinopathy of Prematurity. Br J Ophthalmol July 2009;93(7):986.

12. Mintz-Hittner HA, Kennedy KA, Chuang AZ. Efficacy of intravitreal bevacizumab for stage 3 retinopathy of prematurity. N Engl J Med 2011;364:603-15.

13. American Academy of Pediatrics Section on Ophthalmology; American Academy of Ophthalmology; American Association for Pediatric Ophthalmology and Strabismus; American Association of Certified Orthoptists. Screening examination of premature infants for retinopathy of prematurity. 2013;131(1):189-195

14. Richter GM, Chiang MF et al. Telemedicine for retinopathy of prematurity diagnosis: Evaluation and Challenges. Survey of Ophthalmology Nov-Dec 2009; 54(6):671-684.

15. Skalet $A H$, Quinn GE, Gilbert $C$ et al. Telemedicine screening for Retinopathy of Prematurity in developing countries using digital images: A feasibility project. Journal of AAPOS June 2008; 12(3):252-258.

16. Löfqvist C, Hellstrom A. Validation of a New Retinopathy of Prematurity Screening Method Monitoring Longitudinal Postnatal Weight and Insuline Growth Factor I. Arch Ophthamol 2009; 127 (5): 6622-627.

17. Ann Hellström, Anna-Lena Hård, Eva Engström, AimonNiklasson, Eva Andersson, Lois Smith and ChatarinaLöfqvist. Early Weight Gain Predicts Retinopathy in
Preterm Infants: New, Simple,Efficient Approach to Screening Pediatrics 2009;123;e638- e645; originally published online Mar 16, 2009.

18. Quinn GE, Dobson V, Davitt BV, Hardy RJ, Tung B, Pedroza C, Good WV; Early Treatment for Retinopathy of Prematurity Cooperative Group. Progression of myopia and high myopia in the early treatment for retinopathy of prematurity study: findings to 3 years of age. Ophthalmology Jun 2008 Jun;115(6):10581064.

19. Baker PS, Tasman W. Myopia in Adults with Retinopathy of prematurity. Amer J Ophthal 2008; 145( 6):1090-94

20. Fielder AR, Quinn GE. Myopia of prematurity, nature, nurture or disease?Br J Ophthalmol 1997; 81: 2-3.

21. Davitt BV, Dobson V, Quinn GE, Hardy RJ, Tung B, Good WV; Early Treatment for Retinopathy of Prematurity Cooperative Group. Astigmatism in the Early Treatment for Retinopathy Of Prematurity Study: findings to 3 years of age. Ophthalmology Feb 2009;116(2):332-9.

22. VanderVeen $D$ et al. for the Early treatment of retinopathy of Prematurity Cooperative Group. Prevalence and course of Strabismus in the first Year of life for infants with Prethreshold Retinopathy of Prematurity. ArchivOphthalmol June 2006;124:766-773.

23. O'Keefe M, Kafil-Hussain N, IFlitcroft, Lanigan B. Ocular significance ofintra ventricular haemorrhage in premature infants. Br J Ophthalmol 2001; 85:357-359.

24. O'Connor AR, Wilson CM, Fielder AR. Ophthalmological problems associated with preterm birth. Eye (2007) 21, 1254 1260 